\title{
Exploring the impact of trauma type and extent of exposure on posttraumatic alterations in $5-\mathrm{HT} 1 \mathrm{~A}$ expression
}

\author{
Michael W. Lewis (1)', Russell T. Jones ${ }^{1}$ and Margaret T. Davis (1) ${ }^{2}$
}

\begin{abstract}
The long-term behavioral, psychological, and neurobiological effects of exposure to potentially traumatic events vary within the human population. Studies conducted on trauma-exposed human subjects suggest that differences in trauma type and extent of exposure combine to affect development, maintenance, and treatment of a variety of psychiatric syndromes. The serotonin 1-A receptor (5-HT1A) is an inhibitory G protein-coupled serotonin receptor encoded by the HTR1A gene that plays a role in regulating serotonin release, physiological stress responding, and emotional behavior. Studies from the preclinical and human literature suggest that dysfunctional expression of 5-HT1A is associated with a multitude of psychiatric symptoms commonly seen in trauma-exposed individuals. Here, we synthesize the literature, including numerous preclinical studies, examining differences in alterations in 5-HT1A expression following trauma exposure. Collectively, these findings suggest that the impact of trauma exposure on 5HT1A expression is dependent, in part, on trauma type and extent of exposure. Furthermore, preclinical and human studies suggest that this observation likely applies to additional molecular targets and may help explain variation in trauma-induced changes in behavior and treatment responsivity. In order to understand the neurobiological impact of trauma, including the impact on 5-HT1A expression, it is crucial to consider both trauma type and extent of exposure.
\end{abstract}

\section{Introduction}

Exposure to potentially traumatic events is common, with an estimated rate of over $80 \%$ in the United States and $70 \%$ worldwide ${ }^{1}$. However, long-term behavioral, psychological, and neurobiological effects of exposure vary considerably ${ }^{2}$. Some individuals experience rapid and sustained natural recovery, while others develop chronic trauma-related psychopathology ${ }^{3}$. Importantly, the nature and extent of trauma exposure combine to produce different outcomes; research suggests that exposure to qualitatively different events and different degrees of exposure lead to different psychiatric and neurobiological outcomes $^{4-21}$. For example, qualitatively different events (e.g., rape, assault, and natural disaster) are associated with different levels of conditional risk for posttraumatic

Correspondence: Michael W. Lewis (mlewis1@vt.edu)

${ }^{1}$ Virginia Tech, Blacksburg, VA, USA

${ }^{2}$ Yale University, New Haven, CT, USA

These authors contributed equally: Russell T. Jones, Margaret T. Davis stress disorder (PTSD; 19\% rape and 0.3\% natural disaster $)^{20}$. Similarly, different trauma types are differentially correlated with the emergence of several other psychiatric diagnoses and sequalae following trauma exposure (e.g., depression, anxiety, substance abuse, conduct problems, eating disorders, suicidal ideation, and psychosis $)^{4-19}$. Furthermore, research suggests that genetic risk for PTSD covaries with trauma type, timing, severity, and degree of exposure $^{22-28}$. Critically, research suggests that the dissociative subtype of PTSD and informally recognized subtypes of major depressive disorder (MDD; e.g., anxious depression, and psychotic subtype) may develop as a result of childhood trauma history, are characterized by distinct neurobiological mechanisms, and require different treatment approaches ${ }^{29-34}$. This suggests that common neurobiological factors among trauma-exposed individuals may contribute to heterogeneity in psychiatric symptoms and treatment responsivity; neurobiological phenotypes of trauma-induced psychiatric dysfunction 
transcend traditional diagnostic categories. In order to understand observed variability in the long-term effects of trauma exposure, it may be necessary to examine the relationship between variability in the nature and extent of trauma exposure and associated trauma-induced neurobiological alterations.

Research suggests that individual differences in the expression and activity of serotonin 1-A receptor (5HT1A) are associated with a multitude of psychiatric symptoms commonly seen in trauma-exposed individuals $^{35-40}$. Previous reviews have synthesized literature related to the role of 5-HT1A in depression, anxiety, memory, fear learning, impulsivity, suicide, and social dysfunction ${ }^{35-40}$. In addition, a multitude of preclinical studies have examined trauma-induced alterations in 5$\mathrm{HT}_{1} \mathrm{~A}^{41}$. Though the preclinical literature is consistent in suggesting that trauma alters 5-HT1A expression, specific findings diverge; some studies have observed increasing expression $^{41}$, while others have observed the opposite ${ }^{42}$. In addition, many studies have presented conflicting evidence regarding specific brain regions in which increases and decreases occur ${ }^{41-43}$. Here, we synthesize the literature, including numerous preclinical studies, examining differences in alterations in 5-HT1A expression following trauma exposure. We suggest that observed inconsistencies may be accounted for in part by the influence of differences in the type of trauma subjects are exposed to. Furthermore, we suggest that the same is likely true of other molecular targets and that consideration of trauma type is a key aspect of accurate data interpretation. Thus, we examine the literature on 5-HT1A as an exemplar of a more general observation: in order to understand the neurobiology of trauma, one must account for covariance of trauma type and neurobiological alterations.

\section{5-HT1A}

The 5-HT1A receptor is one of seven inhibitory G protein-coupled serotonin receptors and is encoded by the HTR1A gene ${ }^{44}$. It is found in high density in areas associated with serotonin release (raphe nuclei), memory (hippocampus), fear (amygdala), pleasure (septum), and higher-order cognition (cerebral cortex) ${ }^{38,45-51}$. Physiologically, 5-HT1A activity in areas of high density as well as in several areas of lower density regulates neurotransmitter (e.g., dopamine, acetylcholine, noradrenaline, GABA, and glutamate) and hormone (e.g., cortisol and oxytocin) release as well as neural activity and functional connectivity $^{52-58}$. In addition, 5-HT1A activity has been shown to modulate a wide array of adaptive and maladaptive behaviors. For example, in rodents, the 5-HT1A agonist 8-OH-DPAT has been found to increase impulsive action at low doses, and decrease impulsive action at higher doses ${ }^{59}$. Moreover, both agonists and antagonists of 5-HT1A have been shown to have antidepressant effects in preclinical models ${ }^{60,61}$. The region-specific nature of the physiological effects of 5-HT1A may be relevant to its impact on adaptive and maladaptive behaviors.

Among trauma-exposed individuals, numerous human studies have identified social behaviors as key differentiating factors which separate resilience from vulnerability (e.g., Refs. ${ }^{62,63}$ ). Though it is generally accepted that 5-HT1A exerts strong effects on social behavior, those effects are highly complex ${ }^{64,65}$. For example, one study found that the 5-HT1A full agonist 8-OH-DPAT, the 5-HT1A partial agonist ipsapirone, and the regionspecific partial agonist of 5-HT1A autoreceptors/antagonist of 5-HT1A heteroreceptors MDL-73,005-EF each led to a distinct pattern of effects on social behavior in rodents ${ }^{64}$. Regarding the overall frequency of pro-social behavior, 8-OH-DPAT led to an increase, MDL-73,005EF led to a decrease, and ipsapirone had no effect ${ }^{64}$. In addition to the overall effects, each of the three drugs led to a specific combination of increases and decreases in each of six specific pro-social behaviors, six specific aggressive behaviors, and five specific defensive behaviors $^{64}$. In addition, one study found that treating female marmoset monkeys with the 5-HT1A agonist 8-OHDPAT impaired pair bond quality and decreased female sexual behavior ${ }^{66}$. By contrast, flibanserin, a regionally selective postsynaptic 5-HT1A heteroreceptors agonist/5HT2A antagonist, improved pair bond quality and increased female sexual behavior ${ }^{66}$. Studies such as these have demonstrated the ability of different types of alterations in 5-HT1A activity (as well as its interactions with other receptors) to affect behaviors (both adaptive and maladaptive) shown to develop in the wake of trauma exposure.

In addition to social functioning, 5-HT1A modulates numerous trauma-relevant behaviors. A multitude of preclinical and human studies have linked 5-HT1A expression and activation to a number of well-known trauma sequalae; examples include depression (for reviews, see Refs. ${ }^{35,44,67,68}$ ), anxiety (for reviews, see Refs. ${ }^{36,68}$ ), memory (for reviews, see Refs. ${ }^{38,69-71}$ ), fear learning (for review, see Ref. ${ }^{37}$ ), impulsivity (for review, see Ref. ${ }^{39}$ ), suicide (for review, see Ref. ${ }^{40}$ ), substance abuse (e.g., Ref. ${ }^{72}$ ), social dysfunction (for review, see Ref. ${ }^{64}$ ), and pharmacological treatment responsivity (for reviews, see Refs. ${ }^{73,74}$ ). Given the variety of traumarelated behaviors and neurobiological substrates that 5HT1A receptors modulate, it is reasonable to hypothesize that heterogeneity in trauma-induced 5-HT1A alterations contributes to heterogeneity in psychiatric symptom expression. However, while numerous reviews discuss the impact of 5-HT1A on behaviors which are implicated in trauma, no works of which we are aware have synthesized the literature regarding trauma's impact on 5-HT1A. 
We argue that the importance of synthesizing the literature regarding the relationship between trauma exposure and 5-HT1A expression is that 5-HT1A can act as an exemplar of a general principle: that trauma type, chronicity, and duration likely have an impact on many (and possibly all) trauma-induced molecular alterations. Many of the fundamental mechanisms through which stress alters gene expression in general (e.g., transcription factors, miRNA, etc.) and 5-HT1A expression specifically (e.g., Freud-1 and miR-135a) regulate the expression of multiple genes rather than just one particular gene ${ }^{44}$. Thus, it is unlikely that different types of trauma or degrees of exposure would differentially impact the expression of one gene but not others. Though any number of molecular targets could theoretically be used to demonstrate this principle, 5-HT1A was used in this article for both practical and theorydriven reasons.

Our justification for selecting 5-HT1A as an exemplar here is twofold. First, because 5-HT1A is among the most widely studied molecular mechanisms of stressor-related psychopathology, a robust 5-HT1A focused preclinical literature exists ${ }^{44}$. This is critical because examination of the importance of trauma type and extent of exposure requires comparison of studies for which all other methodologies overlap; only a small portion of the literature meets all of those criteria. Second, by focusing on a molecular target that is widely studied in relation to stressor-related disorders but not often studied in relation to trauma, this work aims to call attention to a possible gap in the literature. In addition to PTSD, we believe that this work has the potential to inform the literature on depression, anxiety, and many other psychiatric diagnoses linked with 5-HT1A dysfunction.

\section{5-HT1A and trauma: human studies}

To date, very few published studies have directly examined the association between trauma exposure and 5-HT1A expression in humans. In addition, none have accounted for trauma type. However, a recent positron emission tomography (PET) study observed a negative correlation between chronic psychosocial stress and 5HT1A availability in vivo in the hippocampus, anterior cingulate cortex, and insular cortex in humans ${ }^{75}$. Furthermore, using PET, subjects with PTSD have been found to have elevated 5-HT1A availability across 13 brain regions ${ }^{76}$. Notably, the results of some PET studies have been shown to vary as a function of which outcome measure researchers employ. For example, when researchers examining the relationship between PTSD and 5-HT1A availability used binding potential nondisplaceable uptake $\left(\mathrm{BP}_{\mathrm{ND}}\right.$; the outcome measure employed in the psychosocial stress study) rather than binding potential free plasma concentration $\left(\mathrm{BP}_{\mathrm{F}}\right)$, no relationship between PTSD and 5-HT1A was observed $^{76,77}$. Another issue that limits interpretation of these findings is the cross-sectional nature of the study designs. Without longitudinal data it is not possible to determine whether dysregulated 5-HT1A expression occurred as a result of stress exposure (traumatic or otherwise) or represents a preexisting risk factor for stressor-induced disorders. For example, elevated 5HT1A availability has been observed in subjects with remitted MDD and has been found to be a heritable preexisting risk factor for $\mathrm{MDD}^{78,79}$. While no published human studies have used longitudinal designs to study the effect of trauma on 5-HT1A expression, a body of preclinical literature suggests that trauma exposure may alter 5-HT1A expression.

\section{Preclinical models of traumatic stress exposure}

In both humans and rodents, the line between nontrauma stress exposure and traumatic stress exposure is often blurry and imprecisely defined. In fact, the "Diagnostic and Statistical Manual" (DSM) definition of trauma exposure has been altered multiple times ${ }^{80}$. Though each DSM has presented a binary definition of PTE exposure, it is unclear whether the present dichotomous approach is adequate $^{80}$. As mentioned previously, qualitatively different types of traumas can produce distinct neurobiological and behavioral consequences (e.g., Ref. ${ }^{21}$ ). In addition, while the DSM uses a binary approach to define the boundary between "stress" and "trauma," it may be important to focus more on qualitative aspects of distinct types of intense stressors and less on a dichotomous variable. For example, both criterion-A-type and noncriterion-A-type childhood maltreatment have been found to be negatively correlated with fractional anisotropy in the inferior longitudinal fasciculus ${ }^{81}$. Controversies regarding the use of a binary definition of trauma, which may incorrectly exclude some extreme stressors while incorrectly amalgamating others, have led to calls for a more fine-grained approach ${ }^{80}$.

The ambiguous and heterogeneous nature of PTEs is reflected in the preclinical trauma literature. A number of different preclinical models have been used to study trauma, including single prolonged stress (SPS), predator threat, restraint stress (RS; aka immobilization stress), inescapable electric shock (IES), fear conditioning (FC), chronic unpredictable stress (CUS; aka chronic variable stress), chronic social defeat (CSD), maternal separation (MS), and MS unpredictable stress. These paradigms and their relationship with trauma have been described and reviewed elsewhere (e.g., Refs. ${ }^{53,82-85}$ ). Of note, CUS is an umbrella term encompassing any paradigm which involves daily exposure to one or more different stressors in a randomized sequence for 1 week or longer ${ }^{86}$. In this work, we only include CUS paradigms that include exposure to at 
least one of the aforementioned acute traumatic stressors. Just as many of the DSM-defined criterion A traumas were not included in the original definition of trauma exposure $^{80}$, most of these preclinical trauma models were not initially conceptualized as representing trauma (e.g., RS, IES, FC, CUS, CSD, and MS) ${ }^{82,83}$. Due to the multifaceted and heterogeneous nature of potentially traumatic events, previous reviews have stated that no single preclinical model of trauma can adequately capture all aspects of trauma and that an ideal approach should draw from a variety of distinct models ${ }^{82,83}$.

While not traditionally viewed as a trauma paradigm, forced swim (FS) has been described as an uncontrollable and anxiogenic life-threatening situation ${ }^{87}$ and has been used in several studies to model trauma-related abnormalities in neurobiology, memory, and pain ${ }^{88-90}$. The behavioral effects of both acute and chronic FS stress have been measured using a number of additional preclinical tests (e.g., sucrose preference test, tail suspension test, elevated plus maze, open field test, social exploration test, Morris water maze, object location test, prepulse inhibition, cocaine preference, ethanol preference) (e.g., Refs. ${ }^{91-110}$ ). Based on those tests, numerous studies have found evidence that FS can induce preclinical dysfunctions reflective of trauma sequalae including anhedonia (e.g., Ref. ${ }^{92}$ ), anxiety (e.g., Ref. ${ }^{110}$ ), social anxiety (e.g., Ref. ${ }^{100}$ ), cognitive dysfunction (e.g., Ref. ${ }^{108}$ ), depression (e.g., Ref. ${ }^{93}$ ), and substance abuse (e.g., Ref. ${ }^{109}$ ). Based on the intense nature of the stressor and its capacity for causing behavioral dysfunctions reflective of multiple facets of trauma-induced psychopathology, FS may represent an additional model of potentially traumatic event exposure. Thus, in order to provide a comprehensive picture of covariance of trauma type and 5-HT1A alterations, FS is included in this review.

Importantly, preclinical trauma models vary qualitatively in ways that make them appropriate analogs of different types of traumatic event exposure. In humans, interpersonal traumas (e.g., physical assault, sexual assault, and family violence) are qualitatively different from non-interpersonal traumas (e.g., severe accident, natural disaster, and life-threatening illness); this contributes to differences in psychiatric symptom severity and outcomes ${ }^{111,112}$. Similarly, some preclinical models incorporate a clear social component (e.g., CSD and MS), while others lack a social component (e.g., FC and RS) ${ }^{83}$. Additional clinically relevant qualitative factors which vary among preclinical trauma models include degree of threat of bodily harm, actual physical pain inflicted, and predictability $^{83}$. In addition to qualitative differences, preclinical trauma models can vary in duration (time per exposure) and chronicity (days of exposure $)^{83}$. In humans, increases in both duration and chronicity of trauma exposure, or number of lifetime exposures to trauma increase subjective peri-traumatic suffering and have been linked to increased symptom severity and qualitatively different psychiatric outcomes ${ }^{112-118}$. Evidence suggests that both qualitative and quantitative differences in rodent trauma models contribute to differences in their impact on rodent behavior and neurobiology ${ }^{83}$. Thus, differences in neurobiological response to various preclinical models of trauma may provide insight into the role of trauma type, duration, and chronicity in contributing to neurobiological heterogeneity among individuals with psychiatric disorders.

\section{Types of traumatic stress exposure and 5-HT1A alterations}

As discussed above, different preclinical paradigms can be seen as analogs of distinct types of traumatic events; preclinical results suggest that when controlling for all other factors, exposure to qualitatively different traumatic events (preclinical paradigms) differentially affects alterations in 5-HT1A mRNA expression. For example, preclinical studies have found that exposure to CUS (approximates chronic exposure to various unpredictable and uncontrollable traumas) led to a decrease in 5-HT1A mRNA expression in all hippocampal subfields compared to controls, but exposure to SPS (approximates single exposure to prolonged perceived life threat) led to a decrease in hippocampal 5-HT1A mRNA expression that was restricted to the CA1 and dentate gyrus ${ }^{42,119}$. In one study, chronic exposure to $20 \mathrm{~min}$ of FS (approximates chronic and predictable exposure to perceived threat of drowning) did not alter 5-HT1A mRNA expression in any hippocampal region ${ }^{119}$. Comparison of these findings reveals that animals exposed to different preclinical paradigms, and thus qualitatively different traumatic events, experienced substantially different alterations in 5HT1A mRNA expression; trauma-induced alterations in hippocampal 5-HT1A mRNA expression may be affected by both quality and frequency of trauma exposure.

Though alterations in mRNA expression represent transcriptional alterations in gene expression, posttranscriptional mechanisms may also cause changes in gene expression, especially during dynamic processes such as those which occur after trauma exposure ${ }^{120}$. Posttranscriptional mechanisms include any process after the transcription of DNA into mRNA, which affect subsequent steps of gene expression ${ }^{120}$. Exposure to SPS, $\mathrm{CF}+$ SPS (SPS preceded by five days of FC), or CUS have led to concurrent increases in 5-HT1A mRNA expression and 5-HT1A expression ${ }^{41,119-122}$. The concurrent nature of these changes in 5-HT1A mRNA and 5-HT1A protein expression suggests that transcriptional mechanisms are at least partially responsible for the observed change in 5HT1A expression in these studies. However, two studies found that exposure to RS (approximates prolonged and 
uncontrollable confinement and threat of bodily harm) or FS (approximates perceived threat of drowning) led to incongruities in 5-HT1A mRNA expression and 5-HT1A expression ${ }^{123,124}$. For example, one study found that exposure to chronic RS led to an increase in 5-HT1A mRNA expression, but a decrease in 5-HT1A expression in the prefrontal cortex ${ }^{123}$. The opposing direction of alterations in 5-HT1A mRNA expression and 5-HT1A expression suggests that posttranscriptional mechanisms led to the decrease in 5-HT1A expression. Furthermore, when controlling for all other factors, one study found that RS and FS exposure led to opposite changes in 5HT1A antagonist binding through posttranscriptional mechanisms; RS led to decreases (CA3 and dentate gyrus), while FS led to increases (CA2 and cortex), though neither exposure affected 5-HT1A mRNA expression ${ }^{124}$. Based on these findings, the degree to which trauma exposure impacts 5-HT1A expression through transcriptional versus posttranscriptional mechanisms may vary by trauma type. Furthermore, when trauma exposure does impact 5HT1A expression through posttranscriptional mechanisms, the direction of change and region affected may also depend upon trauma type. Taken as a whole, this evidence suggests that the impact of trauma exposure on 5-HT1A expression, including the region, direction, and mechanism of change, is influenced by and potentially dependent in part on the type of trauma an individual is exposure to.

Further supporting our proposal that different trauma types may alter 5-HT1A through different mechanisms, research suggests that alterations in the 5 -HT1A repressor Freud-1 may play a role in altering 5-HT1A expression after some, but not all traumas. In one study, exposure to RS led to reduced Freud-1 mRNA and protein in the prefrontal cortex as well as the expected concurrent increase in prefrontal 5-HT1A mRNA in Sprague-Dawley rats $^{123}$. In another study, exposure to CSD (approximates chronic exposure to interpersonal violence) led to decreased prefrontal 5-HT1A mRNA expression in Wistar rats without affecting Freud-1 mRNA expression ${ }^{125}$. Of note, researchers also used different strains of rats (Sprague-Dawley rats and Wistar rats), which may have contributed to observed findings. However, one study which used only one breed of rat examined the effect of nontraumatic stressors in the prefrontal cortex and found that each of four qualitatively different types of stress led to distinct alterations in both mRNA and protein levels of 5-HT1A, Freud-1, and NUDR (NUDR acts as a 5-HT1A autoreceptor repressor and 5-HT1A heteroreceptor enhancer ${ }^{126}$. Overall, this evidence suggests that different types of stress, including different types of traumatic stress, can alter 5-HT1A expression through different mechanisms.

Preclinical pharmacological studies provide additional supporting evidence for our thesis that different types of traumas differentially affect 5-HT1A. Different types of trauma may differentially alter the physiological impact of 5-HT1A activity. One study found that pretreatment with the 5-HT1A agonist ipsapirone differentially affected increases in extracellular levels of adrenocorticotropic releasing hormone, corticosterone, and plasma renin concentration induced by RS, FS, and FC (approximates exposure to predictable physical harm $)^{127}$. In addition, one study found that the 5-HT1A agonist 8-OH-DPAT attenuated the corticosterone response to FC but not RS or IES (approximates exposure to inescapable physical harm $)^{128}$. Based on these results, the observed differential impact of trauma type on 5-HT1A alterations likely has downstream physiological consequences including altered neuro-endocrine functioning.

Though they examine different rodent strains and therefore do not include a direct comparison to other trauma types, additional studies suggest that specific trauma-induced 5-HT1A alterations may have specific behavioral consequences. For example, one study found that, in male Sprague-Dawley rats, SPS led to an increase in 5-HT1A expression in the hippocampal CA1 region as well as impaired spatial memory performance ${ }^{129}$. Local CA1 injection of the 5-HT1A agonist 8-OH-DPAT further exacerbated the SPS-induced impairment, suggesting an impairing effect of SPS-induced 5-HT1A upregulation in the CA1 on spatial memory ${ }^{129}$. By contrast, decreased 5-HT1A expression and activity in the prelimbic ( $\operatorname{PrL})$ cortex may be a critical mechanism of CSD-induced anxiety. One study found that, in female mandarin voles, CSD led to decreased 5-HT1A expression in the PrL, decreased PrL serotonin levels, and increased anxious behavior ${ }^{130}$. Local PrL injection of 8-OH-DPAT reversed the CSD-induced anxiety in exposed voles, while local PrL injection of WAY-100635 (a 5-HT1A antagonist) caused anxious behaviors in control voles ${ }^{130}$. Of note, in CSDexposed voles, 5-HT1A autoreceptor expression was increased in the dorsal raphe nucleus (which projects to the PrL), suggesting that the decreased PrL serotonin levels were caused by a region-specific increase in 5HT1A expression ${ }^{130}$. Overall, these results suggest that region-specific trauma-induced increases and decreases in 5-HT1A expression and activity may have impairing behavioral effects. Given that different types of trauma lead to different region-specific alterations in 5-HT1A, this suggests that the differences in behavioral impact observed in different trauma types may be related to the observed differences in 5-HT1A alterations.

\section{Chronicity and duration of traumatic stress exposure and 5-HT1A alterations}

As is the case with different types of traumas, preclinical results suggest that, when controlling for all other factors, differences in duration (time per exposure) or chronicity 
(days of exposure) lead to different alterations in 5-HT1A expression and 5-HT1A mRNA expression. For example, in one study, exposure to $30 \mathrm{~min}$ of FS led to an increase in 5-HT1A agonist binding and 5-HT1A antagonist binding in the hippocampus and cortex ${ }^{124}$. By contrast, exposure to only $15 \mathrm{~min}$ of FS did not alter 5-HT1A agonist binding or 5-HT1A antagonist binding in the hippocampus or cortex ${ }^{124}$. Similarly, findings from two studies suggest that exposure to 14 days of CUS led to a decrease in hippocampal 5-HT1A mRNA expression, but exposure to 7 days of the same CUS paradigm did not alter hippocampal 5-HT1A mRNA expression ${ }^{42,119}$. As the extent of trauma exposure leads to different clinical consequence in humans, preclinical evidence suggests that both duration and chronicity of exposure appear to impact 5-HT1A expression.

Differences in chronicity of trauma exposure can differentially impact 5-HT1A expression in complex ways. For example, in one study, exposure to CSD led to a transient increase in 5-HT1A binding in the claustrum, which was found when exposure lasted 2 days, but not when exposure lasted 10 or more days ${ }^{43}$. However, exposure to the same CSD paradigm led to a stable decrease in 5-HT1A binding in the posterior cingulate, which was found when exposure lasted 10, 21, or 28 days $^{43}$. Exposure for 28 days also led to decreases in the parietal cortex, prefrontal cortex, regio retrobulbaris, and CA1 hippocampal region, which were not found when exposure lasted $<28$ days $^{43}$. These results support a possible region-specific dose-response relationship between chronicity of trauma exposure and 5-HT1A binding. Interestingly, no change was detected in the raphe for any chronicity of CSD up to 28 days ${ }^{43}$. In another study, $2 \mathrm{~h}$ of exposure to RS led to an increase in hippocampal 5-HT1A binding when exposure lasted 1 day and a further increase in 5 -HT1A binding when exposure lasted 5 days ${ }^{131}$. In contrast with the overall increase in hippocampal 5-HT1A binding observed between 1 and 5 days of exposure, increases in the CA4 subregion were transient; increases in 5-HT1A binding in the CA4 were observed when exposure lasted for 1 day but not when it lasted for 5 days ${ }^{131}$. Overall, findings suggest that changes in length of exposure may lead to distinct region-specific changes in the degree and direction of the relationship between trauma exposure and 5-HT1A expression; these changes can be transient in nature or delayed in onset. Taken together, preclinical findings support the assertion that differences in qualitative and quantitative aspects of trauma exposure contribute to variation in subsequent alterations in 5-HT1A expression.

\section{Clinical/pharmacological implications}

The exact role of 5-HT1A in the etiology of traumarelated psychiatric disorders is unclear. However, the prevailing consensus is that $5-\mathrm{HT} 1 \mathrm{~A}$ is influential in the development of depression, anxiety, fear, and memory impairment ${ }^{35-37}$. In addition, one study finds that individuals with PTSD may have elevated 5-HT1A availability ${ }^{76}$. Further elucidation of 5-HT1A's behavioral impact, including the degree to which it varies as a function of trauma history, may lead to the development of highly targeted treatments, and betterment of outcomes through personalized treatment ${ }^{44,132}$. For example, preclinical studies suggest that biased 5-HT1A agonists, which specifically target autoreceptor or heteroreceptor signaling, may be more effective in treating depression and anxiety than currently available 5-HT1A agonists ${ }^{133-135}$. In addition, recent literature suggests that drugs capable of acting on specific mechanisms of 5-HT1A expression could lead to major breakthroughs in treating depression ${ }^{44}$. However, targeting the correct mechanism for alteration of 5-HT1A may be critical ${ }^{4,136}$. Given the impact of trauma type and extent of exposure on $5-\mathrm{HT} 1 \mathrm{~A}$, we theorize that trauma history may be a crucial determinant of response to future breakthrough treatments.

In support of our theory, previous correlational studies and randomized controlled trials have found evidence to suggest that individuals with the same diagnosis but different trauma histories respond differently to currently available pharmacological treatments ${ }^{137-144}$. Thus, individuals with different trauma histories may require different pharmacological treatments, likely in part due to the differential effects of those trauma histories on gene expression. Failure to account for trauma history during pharmacological studies may obscure trauma-induced heterogeneity in responsivity, thus concealing a drug's potential efficacy (or lack thereof) in individuals with particular trauma histories and delaying progress in clinical practice. Based on this evidence, in order to achieve personalized treatment, one must first account for the differential impact of different types of trauma and degrees of exposure.

\section{5-HT1A as an exemplar}

Though the primary focus of this review has been 5HT1A, the implications of these findings likely extend to additional molecular targets. Multiple preclinical studies suggest that different types of trauma and degrees of exposure can lead to alterations in molecular targets other than 5-HT1A. For example, exposure to CUS led to multiple region-specific increases and decreases in dopamine D1-like receptor binding, but exposure to RS did not impact D1-like receptor binding in any of those regions ${ }^{145}$. Similarly, in the hippocampal CA2, exposure to RS led to an increase in serotonin-7 receptor mRNA expression but exposure to CUS did not ${ }^{146}$. Additional examples exist, including research focused on mu opioid and glucocorticoid receptors ${ }^{147,148}$. Similarly, multiple 
studies have found evidence to suggest that different types of trauma exposure and different degrees of trauma exposure differentially affect gene expression in humans ${ }^{149-153}$. For example, in one study, profiles of gene expression in peripheral blood cells which distinguished individuals with PTSD from controls were almost completely distinct (98\% nonoverlapping), depending on whether subjects with PTSD had a history of childhood abuse exposure ${ }^{153}$. Importantly, in addition to PTSD, the impact of trauma type and degree of exposure on gene expression has been evident in individuals with MDD and borderline personality disorder ${ }^{150}$. Taken together, these studies provide direct evidence for the importance of trauma type and degree of exposure in modulating the effect of trauma on genetic expression, which extends beyond 5-HT1A and beyond PTSD.

Previously, we synthesized evidence to suggest that different types of traumas can alter 5-HT1A through different mechanisms, including the 5-HT1A repressor Freud-1. A closer examination of specific mechanisms of 5-HT1A alterations provides a useful paradigm for understanding the complexity of the concurrent impact of different trauma types and degrees of exposure on many molecular mechanisms. For example, the 5-HT1A repressor Freud-1 also represses the dopamine receptor $\mathrm{D}_{2}{ }^{154}$. Thus, differences in the impact of various types of trauma on Freud-1 likely lead to differences in expression of not only 5-HT1A, but also $\mathrm{D}_{2}$ and any other mechanisms affected by Freud-1. In addition, we provided evidence that different types of stressors differentially impact the dual-effect 5-HT1A autoreceptor repressor and 5-HT1A heteroreceptor enhancer NUDR. In addition to its effect on 5-HT1A, NUDR also regulates transcription of Proenkephalin ${ }^{155}$. Notably, many transcriptional and posttranscriptional mechanisms of 5-HT1A expression also affect multiple molecular targets ${ }^{44}$. For example, the 5-HT1A autoreceptor enhancer Pet-1 also plays a role in activating expression of the nicotinic acetylcholine receptor ${ }^{156,157}$. In addition, 5-HT1A promotors MAZ and Sp1 decrease NMDA receptor subunit type 1 promoter activity $^{158,159}$. Furthermore, in addition to posttranscriptional regulation of 5-HT1A, miR-135a also regulates the serotonin transporter, PHLPP2, and FOXO1 ${ }^{44}$. These findings illustrate the general principal that the mechanisms underlying differential impacts of trauma type and chronicity of exposure on 5-HT1A expression likely lead to differential impacts on other molecular targets as well. Furthermore, they provide insight into the fact that traumatic stressors may regulate a wide array of molecular targets through diffusely acting mechanisms.

\section{Limitations and future directions}

This paper does not provide an exhaustive review of the literature on 5-HT1A and trauma. Many studies which found alterations in 5-HT1A expression induced by rodent trauma models were excluded from mention in this work due to a lack of direct comparability arising from methodological discrepancies ${ }^{58,160-184}$. Future preclinical studies can further elucidate the impact of differences in trauma type and extent of exposure on changes in the expression of 5-HT1A and other molecular targets by consciously and deliberately accounting for trauma type. One particularly noteworthy gap in the current literature concerns early life trauma. To date, models of early life trauma have been associated with increases ${ }^{183}$, decreases ${ }^{174}$, or no change ${ }^{184}$ in 5-HT1A expression. However, methodological differences (e.g., rodent strain, rodent sex, chronicity of exposure, additional experimental stressors, time since trauma exposure at sacrifice, and index of 5-HT1A expression) preclude direct comparisons between trauma models or between different developmental periods as they relate to 5-HT1A expression. Future studies should endeavor to resolve this issue.

More studies are needed in order to identify relationships between specific categories of trauma and resultant alterations in gene expression. In addition, these findings have yet to be extended to humans. Only one published study in humans has evaluated the relationship between 5-HT1A and trauma exposure, with null results ${ }^{185}$. Of note, however, trauma type was not controlled for and authors acknowledged that the study may have lacked sufficient power to detect an association $^{185}$. Future studies should examine the association between trauma and 5-HT1A expression in humans, specifically with respect to differences in trauma type and chronicity of exposure. Given the known adverse impacts of aversive childhood experiences and the divergence in genetic expression associated with childhood and adult trauma, differences in developmental timing of trauma should also be considered.

While it seems clear based on the reviewed preclinical literature that trauma type and chronicity of exposure impact gene expression, no available preclinical studies examining 5-HT1A distinguish behaviorally affected and resilient individuals. In the preclinical literature, the importance of separating behaviorally affected animals from resilient animals is becoming increasingly clear ${ }^{82,83}$. Studies have found that some post-trauma alterations in gene expression that are present in the group average may only apply to affected animals, while others may only apply to resilient animals ${ }^{82,83}$. Thus, with the exception of studies that measure the behavioral effects of 5-HT1A agonists or antagonists in addition to measuring gene expression, most currently available studies do not provide information to distinguish adaptive alterations in 5HT1A from maladaptive alterations. Future studies should apply methodology such as cut-off behavioral criteria and behavioral profiling in order to distinguish 
alterations in 5-HT1A that are seen in resilient animals from those seen in affected animals. Of note, cut-off behavioral criteria and behavioral profiling are applied analytically to postexposure behavioral data and do not necessarily require a change in study design ${ }^{82,83}$. Thus, it may be possible to apply these techniques to archival data. Though some research questions may require the design of new studies in order to incorporate specific behavioral effects of interest, we recommend applying cut-off behavioral criteria and/or behavioral profiling to archival data wherever feasible. In addition to being highly practical, the use of archival data is in line with ethical best practice as it does not cause any additional suffering to animals ${ }^{186}$.

This review focuses on the importance of considering differences in the nature and chronicity of trauma exposure in neurobiological research. However, other key variables warrant similar consideration. Specifically, recent research has emphasized the importance of sex differences, risk factors, and individual differences in response to the same trauma ${ }^{82}$. We propose that these two approaches need not be mutually exclusive. For example, in rodents, qualitatively different types of nontraumatic stressors have been found to lead to sex-dependent differential alterations in mRNA and density of 5-HT1A and specific 5 -HT1A transcription factors ${ }^{126}$. Future studies should examine the degree to which the interaction of stressor type and sex extends to rodent trauma models and human trauma exposure. This approach could also be extended to other relevant risk factors, such as inherited genetic differences, which are shown to alter trauma's impact on 5-HT1A expression as well as behavior ${ }^{182}$. Specific single nucleotide polymorphisms alter the ability of specific mechanisms to impact gene expression ${ }^{187}$. Specific mechanisms underlying the relationship between trauma and gene expression depend on trauma type and level of exposure. Therefore, future studies should investigate specific interactions between genes, sex, and trauma type as they relate to 5 -HT1A expression.

\section{Conclusions}

We have presented preclinical evidence supporting our contention that differences in the type and chronicity of exposure to trauma leads to differences in the regionspecific posttraumatic alterations in 5 -HT1A. Furthermore, differences in the nature of trauma and extent of exposure to trauma appear to lead to differences in the mechanisms underlying changes in 5-HT1A expression; even when two different traumas lead to the same directional change in 5-HT1A expression in a given region, it is possible that different mechanisms are driving the change. Furthermore, this phenomenon does not appear to be specific to 5-HT1A and evidence suggests that it may translate to humans. Based on the evidence presented in this review, future studies aimed at understanding the differential molecular alterations that are generally associated with certain categories of trauma exposure may eventually inform a more targeted approach to pharmacological treatments for traumaexposed individuals. In order to understand the neurobiology of trauma, it is crucial to consider both trauma type and extent of exposure.

\section{Acknowledgements}

The authors would like to thank Maddy Ryan for her assistance with this paper.

\section{Conflict of interest}

The authors declare that they have no conflict of interest.

\section{Publisher's note}

Springer Nature remains neutral with regard to jurisdictional claims in published maps and institutional affiliations.

Received: 20 November 2019 Revised: 15 June 2020 Accepted: 23 June 2020

Published online: 16 July 2020

\section{References}

1. Benjet, $\mathrm{C}$. et al. The epidemiology of traumatic event exposure worldwide: results from the World Mental Health Survey Consortium. Psychol. Med. 46, 327-343 (2016).

2. Guina, J. et al. Should posttraumatic stress be a disorder or a specifier? Towards improved nosology within the DSM categorical classification system. Curr. Psychiatry Rep. 19, 66 (2017).

3. Bonanno, G. A. \& Mancini, A. D. Beyond resilience and PTSD: mapping the heterogeneity of responses to potential trauma. Psychol. Trauma 4, 74 (2012).

4. Krupnick, J. L. et al. Mental health effects of adolescent trauma exposure in a female college sample: exploring differential outcomes based on experiences of unique trauma types and dimensions. Psychiatry 67, 264-279. (2004).

5. Wanklyn, S. G. et al. Trauma types as differential predictors of posttraumatic stress disorder (PTSD), major depressive disorder (MDD), and their comorbidity. Can. J. Behav. Sci. 48, 296 (2016).

6. Briggs-Gowan, M. J. et al. Exposure to potentially traumatic events in early childhood: differential links to emergent psychopathology. J. Child Psychol. Psychiatry 51, 1132-1140 (2010).

7. Freeman, D. \& Fowler, D. Routes to psychotic symptoms: trauma, anxiety and psychosis-like experiences. Psychiatry Res. 169, 107-112 (2009).

8. Hall, A., Kimberly, A., Bartlett, B. A., Iverson, K. M. \& Mitchell, K. S. Eating disorder symptoms in female veterans: the role of childhood, adult, and military trauma exposure. Psychol. Trauma 10, 345 (2018).

9. Khan, A. et al. Examining the impact of different types of military trauma on suicidality in women veterans. Psychiatry Res. 274, 7-11 (2019).

10. Goldsmith, R. E., Freyd, J. J. \& DePrince, A. P. Betrayal trauma: associations with psychological and physical symptoms in young adults. J. Interpers. Violence 27, 547-567 (2012).

11. Briere, J. \& Runtz, M. Differential adult symptomatology associated with three types of child abuse histories. Child Abuse Negl. 14, 357-364 (1990).

12. Sullivan, T. P., Fehon, D. C., Andres-Hyman, R. C., Lipschitz, D. S. \& Grilo, C. M. Differential relationships of childhood abuse and neglect subtypes to PTSD symptom clusters among adolescent inpatients. J. Trauma Stress. 19, 229-239 (2006).

13. Prigerson, H. G., Maciejewski, P. K. \& Rosenheck, R. A. Combat trauma: trauma with highest risk of delayed onset and unresolved posttraumatic stress disorder symptoms, unemployment, and abuse among men. J. Nerv. Ment. Dis. 189, 99-108 (2001).

14. Amir, M., Kaplan, Z. \& Kotler, M. Type of trauma, severity of posttraumatic stress disorder core symptoms, and associated features. J. Gen. Psychol. 123, 341-351 (1996). 
15. Ford, J. D., Stockton, P., Kaltman, S. \& Green, B. L. Disorders of extreme stress (DESNOS) symptoms are associated with type and severity of interpersonal trauma exposure in a sample of healthy young women. J. Interpers. Violence 21, 1399-1416 (2006)

16. Smith, H. L., Summers, B. J., Dillon, K. H. \& Cougle, J. R. Is worst-event trauma type related to PTSD symptom presentation and associated features? J. Anxiety Disord. 38, 55-61 (2016).

17. Contractor, A. A., Caldas, S. V., Dolan, M., Lagdon, S. \& Armour, C. PTSD's factor structure and measurement invariance across subgroups with differing count of trauma types. Psychiatry Res. 264, 76-84 (2018).

18. Hyland, P. et al. Variation in post-traumatic response: the role of trauma type in predicting ICD-11 PTSD and CPTSD symptoms. Soc. Psychiatry Psychiatr. Epidemiol. 52, 727-736 (2017)

19. Kilpatrick, D. G. et al. National estimates of exposure to traumatic events and PTSD prevalence using DSM-IV and DSM-5 criteria. J. Trauma Stress 26 537-547 (2013).

20. Kessler, R. C. et al. Trauma and PTSD in the WHO world mental health surveys. Eur. J. Psychotraumatol. 8, 1353383 (2017).

21. Cassiers, L. L. M. et al. Structural and functional brain abnormalities associated with exposure to different childhood trauma subtypes: a systematic review of neuroimaging findings. Front. Psychiatry 9, 329 (2018).

22. Cornelis, M. C., Nugent, N. R., Amstadter, A. B. \& Koenen, K. C. Genetics of post-traumatic stress disorder: review and recommendations for genome-wide association studies. Curr. Psychiatry Rep. 12, 313-326 (2010).

23. Kolassa, I.-T., Kolassa, S., Ertl, V., Papassotiropoulos, A. \& Dominique, J.-F. The risk of posttraumatic stress disorder after trauma depends on traumatic load and the catechol-O-methyltransferase Val158Met polymorphism. Biol. Psychiatry 67, 304-308 (2010).

24. Mehta, D. \& Binder, E. B. Genex environment vulnerability factors for PTSD: the HPA-axis. Neuropharmacology 62, 654-662 (2012).

25. Zannas, A. \& Binder, E. Gene-environment interactions at the FKBP5 locus: sensitive periods, mechanisms and pleiotropism. Genes Brain Behav. 13, 25-37 (2014).

26. Huckins, L. M. et al. Analysis of Genetically Regulated Gene Expression Identifies a Prefrontal PTSD Gene, SNRNP35, Specific to Military Cohorts. Cell Reports https://doi.org/10.1016/j.celrep.2020.107716 (2020).

27. Nievergelt, C. et al. 157. Large-scale genetic characterization of PTSD: addressing heterogeneity across ancestry, sex, and trauma. Biol. Psychiatry 83, S64 (2018)

28. Miller, M. et al. CRP polymorphisms and DNA methylation of the AIM2 gene influence associations between trauma exposure, PTSD, and C-reactive protein. Brain Behav. Immun. 67, 194-202 (2018).

29. Heim, C., Newport, D. J., Mletzko, T., Miller, A. H. \& Nemeroff, C. B. The link between childhood trauma and depression: insights from HPA axis studies in humans. Psychoneuroendocrinology 33, 693-710 (2008).

30. Lanius, R. A., Brand, B., Vermetten, E., Frewen, P. A. \& Spiegel, D. The dissociative subtype of posttraumatic stress disorder: rationale, clinical and neurobiological evidence, and implications. Depress. Anxiety 29, 701-708 (2012).

31. Menke, A. et al. Childhood trauma dependent anxious depression sensitizes HPA axis function. Psychoneuroendocrinology 98, 22-29 (2018).

32. Tokuda, T. et al. Identification of depression subtypes and relevant brain regions using a data-driven approach. Sci. Rep. 8, 14082 (2018).

33. Gaudiano, B. A. \& Zimmerman, M. The relationship between childhood trauma history and the psychotic subtype of major depression. Acta Psychiatr. Scand. 121, 462-470 (2010).

34. Croarkin, P. E. Indexing the neurobiology of psychotic depression with resting state connectivity: insights from the STOP-PD study. EBioMedicine 37, 32-33 (2018).

35. Kaufman, J., DeLorenzo, C., Choudhury, S. \& Parsey, R. The 5-HT1A receptor in major depressive disorder. Eur. Neuropsychopharmacol. 26, 397-410 (2016).

36. Garcia-Garcia, A. L., Newman-Tancredi, A. \& Leonardo, E. D. P5-HT 1A receptors in mood and anxiety: recent insights into autoreceptor versus heteroreceptor function. Psychopharmacology 231, 623-636 (2014).

37. Ögren, S. O. et al. The role of 5-HT 1A receptors in learning and memory. Behav. Brain Res. 195, 54-77 (2008).

38. Glikmann-Johnston, Y., Saling, M. M., Reutens, D. C. \& Stout, J. C. Hippocampal 5-HT1A receptor and spatial learning and memory. Front. Pharmacol. 6, 289 (2015).
39. Pattij, T. \& Schoffelmeer, A. N. Serotonin and inhibitory response control: focusing on the role of 5-HT1A receptors. Eur. J. Pharmacol. 753, 140-145 (2015).

40. Mann, J. J., Arango, V., Marzuk, P. M., Theccanat, S. \& Reis, D. Evidence for the 5-HT hypothesis of suicide a review of post-mortem studies. Br. J. Psychiatry 155, 7-14 (1989).

41. Luo, F. F., Han, F. \& Shi, Y. X. Changes in 5-HT1A receptor in the dorsal raphe nucleus in a rat model of post-traumatic stress disorder. Mol. Med. Rep. 4, 843-847 (2011).

42. López, J. F., Liberzon, I., Vázquez, D. M., Young, E. A. \& Watson, S. J. Serotonin $1 \mathrm{~A}$ receptor messenger RNA regulation in the hippocampus after acute stress. Biol. Psychiatry 45, 934-937 (1999).

43. Flùgge, G. Dynamics of central nervous 5-HT1A-receptors under psychosocial stress. J. Neurosci. 15, 7132-7140 (1995).

44. Albert, P. R., Le François, B. \& Vahid-Ansari, F. Genetic, epigenetic and posttranscriptional mechanisms for treatment of major depression: the 5-HT1A receptor gene as a paradigm. J. Psychiatry Neurosci. 44, 164 (2019).

45. Ito, H., Halldin, C. \& Farde, L. Localization of 5-HT1A receptors in the living human brain using [carbonyl-11C] WAY-100635: PET with anatomic standardization technique. J. Nucl. Med. 40, 102-109 (1999).

46. Kia, H. K., Brisorgueil, M. J., Hamon, M., Calas, A. \& Vergé, D. Ultrastructural localization of 5-hydroxytryptamine1A receptors in the rat brain. J. Neurosci. Res. 46, 697-708 (1996).

47. Hall, H. et al. Autoradiographic localization of 5-HT1A receptors in the postmortem human brain using [3H] WAY-100635 and [11C] way-100635. Brain Res. 745, 96-108 (1997).

48. Weissmann-Nanopoulos, D., Mach, E., Magre, J., Demassey, Y. \& Pujol, J.-F. Evidence for the localization of $5 \mathrm{HT} 1 \mathrm{~A}$ binding sites on serotonin containing neurons in the raphe dorsalis and raphe centralis nuclei of the rat brain. Neurochem. Int. 7, 1061-1072 (1985).

49. $\mathrm{Li}, \mathrm{X}$. et al. 5-HT1A receptor agonist affects fear conditioning through stimulations of the postsynaptic 5-HT1A receptors in the hippocampus and amygdala. Eur. J. Pharmacol. 532, 74-80 (2006).

50. Knapp, C. M. \& Kornetsky, C. Neural basis of pleasure and reward. Handbook of Neuroscience for the Behavioral Sciences, Vol. 2, 781-806 (John Wiley \& Sons, 2009).

51. Depoortère, R. et al. F15599, a preferential post-synaptic 5-HT1A receptor agonist: activity in models of cognition in comparison with reference 5-HT1A receptor agonists. Eur. Neuropsychopharmacol. 20, 641-654 (2010).

52. Li, Q. et al. Medial hypothalamic 5-hydroxytryptamine (5-HT) 1A receptors regulate neuroendocrine responses to stress and exploratory locomotor activity: application of recombinant adenovirus containing 5-HT1A sequences. J. Neurosci. 24, 10868-10877 (2004).

53. Razoux, F. et al. Transgenerational disruption of functional 5-HT 1A R-induced connectivity in the adult mouse brain by traumatic stress in early life. Mol. Psychiatry 22, 519 (2017).

54. Fink, K. B. \& Göthert, M. 5-HT receptor regulation of neurotransmitter release. Pharmacol. Rev. 59, 360-417 (2007).

55. Rojas, P. S. \& Fiedler, J. L. What do we really know about 5-HT1A receptor signaling in neuronal cells? Front. Cell Neurosci. 10, 272 (2016).

56. Osei-Owusu, P., James, A., Crane, J. \& Scrogin, K. E. 5-Hydroxytryptamine 1A receptors in the paraventricular nucleus of the hypothalamus mediate oxytocin and adrenocorticotropin hormone release and some behavioral components of the serotonin syndrome. J. Pharmacol. Exp. Ther. 313, 1324-1330 (2005).

57. Choi, I.-S., Cho, J.-H. \& Jang, I.-S. 5-Hydroxytryptamine 1 A receptors inhibit glutamate release in rat medullary dorsal horn neurons. Neuroreport 24, 399-403 (2013).

58. Harvey, B. H., Naciti, C., Brand, L. \& Stein, D. J. Endocrine, cognitive and hippocampal/cortical 5HT1A/2A receptor changes evoked by a timedependent sensitisation (TDS) stress model in rats. Brain Res. 983, 97-107 (2003).

59. Poulos, C. X., Parker, J. \& Le, A. Dexfenfluramine and 8-OH-DPAT modulate impulsivity in a delay-of-reward paradigm: implications for a correspondence with alcohol consumption. Behav. Pharmacol. 7, 395-399 (1996).

60. Celada, P., Puig, M. V., Amargós-Bosch, M., Adell, A. \& Artigas, F. The therapeutic role of 5-HT1A and 5-HT2A receptors in depression. J. Psychiatry Neurosci. 29, 252 (2004).

61. Blier, P. \& Ward, N. M. Is there a role for $5-\mathrm{HT} 1 \mathrm{~A}$ agonists in the treatment of depression? Biol. Psychiatry 53, 193-203 (2003). 
62. Charuvastra, A. \& Cloitre, M. Social bonds and posttraumatic stress disorder. Annu. Rev. Psychol. 59, 301-328 (2008).

63. Davis, M. T., Witte, T. K. \& Weathers, F. W. Posttraumatic stress disorder and suicidal ideation: the role of specific symptoms within the framework of the interpersonal-psychological theory of suicide. Psychol. Trauma 6, 610 (2014).

64. Bell, R. \& Hobson, H. 5-HT1A receptor influences on rodent social and agonistic behavior: a review and empirical study. Neurosci. Biobehav. Rev. 18, 325-338 (1994).

65. de Boer, S. F. \& Koolhaas, J. M. 5-HT1A and 5-HT1B receptor agonists and aggression: a pharmacological challenge of the serotonin deficiency hypothesis. Eur. J. Pharmacol. 526, 125-139 (2005).

66. Aubert, Y. et al. Flibanserin and 8-OH-DPAT implicate serotonin in association between female marmoset monkey sexual behavior and changes in pairbond quality. J. Sex. Med. 9, 694-707 (2012).

67. Albert, P. R. \& Le François, B. Modifying 5-HT1A receptor gene expression as a new target for antidepressant therapy. Front. Neurosci. 4, 35 (2010).

68. Albert, P. R. \& Fiori, L. M. Transcriptional dys-regulation in anxiety and major depression: 5-HT1A gene promoter architecture as a therapeutic opportunity. Curr. Pham. Des. 20, 3738-3750 (2014).

69. Meneses, A. \& Perez-Garcia, G. 5-HT1A receptors and memory. Neurosci. Biobehav. Rev. 31, 705-727 (2007).

70. Ögren, S. O. et al. The role of 5-HT1A receptors in learning and memory. Behav. Brain Res. 195, 54-77 (2008).

71. Bert, B., Fink, H., Rothe, J., Walstab, J. \& Bönisch, H. Learning and memory in 5HT1A-receptor mutant mice. Behav. Brain Res. 195, 78-85 (2008).

72. Tomkins, D. M., Sellers, E. M. \& Fletcher, P. Median and dorsal raphe injections of the 5-HT1A agonist, 8-OH-DPAT, and the GABAA agonist, muscimol, increase voluntary ethanol intake in Wistar rats. Neuropharmacology 33, 349-358 (1994).

73. Cryan, J. F. \& Leonard, B. E. 5-HT1A and beyond: the role of serotonin and its receptors in depression and the antidepressant response. Hum. Psychopharmacol. 15, 113-135 (2000).

74. Sniecikowska, J., Newman-Tancredi, A. \& Kolaczkowski, M. From receptor selectivity to functional selectivity: the rise of biased agonism in 5-HT1A receptor drug discovery. Curr. Top. Med. Chem. 19, 2393-2420 (2019).

75. Jovanovic, H., Perski, A., Berglund, H. \& Savic, I. Chronic stress is linked to 5HT1A receptor changes and functional disintegration of the limbic networks. Neuroimage 55, 1178-1188 (2011).

76. Sullivan, G. M. et al. Higher in vivo serotonin-1a binding in posttraumatic stress disorder: a PET study with [11C] WAY-100635. Depress. Anxiety 30 197-206 (2013).

77. Bonne, O. et al. No change in serotonin type $1 \mathrm{~A}$ receptor binding in patients with posttraumatic stress disorder. Am. J. Psychiatry 162, 383-385 (2005).

78. Milak, M. S. et al. Higher 5-HT1A autoreceptor binding as an endophenotype for major depressive disorder identified in high risk offspring - a pilot study. Psychiatry Res. 276, 15-23 (2018).

79. Miller, J. M. et al. Elevated serotonin $1 \mathrm{~A}$ binding in remitted major depressive disorder: evidence for a trait biological abnormality. Biol. Psychiatry 34, 2275 (2009).

80. Stein, J. Y., Wilmot, D. V. \& Solomon, Z. Does one size fit all? Nosological, clinical, and scientific implications of variations in PTSD Criterion A. J. Anxiety Disord. 43, 106-117 (2016).

81. Olson, E. A. et al. Childhood maltreatment experiences are associated with altered diffusion in occipito-temporal white matter pathways. Brain Behav. 10, e01485 (2019).

82. Richter-Levin, G., Stork, O. \& Schmidt, M. V. Animal models of PTSD: a challenge to be met. Mol. Psychiatry 1, 1135-1156 (2018).

83. Whitaker, A. M., Gilpin, N. W. \& Edwards, S. Animal models of post-traumatic stress disorder and recent neurobiological insights. Behav. Pharmacol. 25, 398 (2014).

84. Yamamoto, S. et al. Single prolonged stress: toward an animal model of posttraumatic stress disorder. Depress. Anxiety 26, 1110-1117 (2009).

85. Wilson, C. B., Ebenezer, P. J., McLaughlin, L. D. \& Francis, J. Predator exposure/ psychosocial stress animal model of post-traumatic stress disorder modulates neurotransmitters in the rat hippocampus and prefrontal cortex. PLOS ONE $\mathbf{9}$, e89104 (2014).

86. Wilson, M. A., Grillo, C. A., Fadel, J. R. \& Reagan, L. P. Stress as a one-armed bandit: Differential effects of stress paradigms on the morphology, neurochemistry and behavior in the rodent amygdala. Neurobiol. Stress $\mathbf{1}$ 195-208 (2015).
87. Almeida, P., Trovo, M., Tokumoto, A., Pereira, A. \& Padovan, C. Role of serotonin $1 \mathrm{~A}$ receptors in the median raphe nucleus on the behavioral consequences of forced swim stress. J. Psychopharmacol. 27, 1134-1140 (2013).

88. Ježek, K. et al. Stress-induced out-of-context activation of memory. PLoS Biol. 8, 12 (2010).

89. Niknazar, S. et al. Effect of maternal stress prior to conception on hippocampal BDNF signaling in rat offspring. Mol. Neurobiol. 54, 6436-45. (2017).

90. Linnstaedt, S. D. et al. Genetic variant rs3750625 in the $3^{\prime}$ UTR of ADRA2A affects stress-dependent acute pain severity after trauma and alters a microRNA-34a regulatory site. Pain 158, 230 (2017).

91. Serchov, T. et al. Increased signaling via adenosine A1 receptors, sleep deprivation, imipramine, and ketamine inhibit depressive-like behavior via induction of Homer1a. Neuron 87, 549-562 (2015).

92. Gomez, J., Haas, N. A. \& Schwarz, J. M. An IL-6 receptor antagonist attenuates postpartum anhedonia, but has no effect on anhedonia precipitated by subchronic stress in female rats. Psychopharmacology 236, 2983-2995 (2019).

93. Qi, X., Lin, W., Li, J., Pan, Y. \& Wang, W. The depressive-like behaviors are correlated with decreased phosphorylation of mitogen-activated protein kinases in rat brain following chronic forced swim stress. Behav. Brain Res. 175, 233-240 (2006).

94. Chen, L. et al. Activation of CRF/CRFR1 signaling in the basolateral nucleus of the amygdala contributes to chronic forced swim-induced depressive-like behaviors in rats. Behav. Brain Res. 338, 134-142 (2018).

95. Qi, X. et al. Fluoxetine increases the activity of the ERK-CREB signal system and alleviates the depressive-like behavior in rats exposed to chronic forced swim stress. Neurobiol. Dis. 31, 278-285 (2008).

96. Liu, R. et al. Citalopram alleviates chronic stress induced depression-like behaviors in rats by activating GSK3 $\beta$ signaling in dorsal hippocampus. Brain Res. 1467, 10-17 (2012).

97. Pan, Y. et al. The effects of central pro-and anti-inflammatory immune challenges on depressive-like behavior induced by chronic forced swim stress in rats. Behav. Brain Res. 247, 232-240 (2013).

98. Bai, Y., Zhang, Y., Jiang, S., Zheng, X. \& Liu, Z. The effects of acute stress on consummatory and motivational responses for sucrose in rats after longterm withdrawal from morphine. Psychopharmacology 236, 3197-208. (2019).

99. Kaur, G. \& Kulkarni, S. Differential effect of a polyherbal formulation-OB-200G in male and female mice subjected to forced swim stress. Indian J. Physiol. Pharmacol. 44, 281-289 (2000).

100. Christianson, J. P., Drugan, R. C., Flyer, J. G., Watkins, L. R. \& Maier, S. F. Anxiogenic effects of brief swim stress are sensitive to stress history. Prog. Neuropsychopharmacol. Biol. Psychiatry 44, 17-22 (2013).

101. Bernal-Morales, B., Guillén-Ruiz, G., Cueto-Escobedo, J., Rodríguez-Landa, J. F. \& Contreras, C. M. Sensitivity to diazepam after a single session of forced swim stress in weaning Wistar rats. Acta Pharm. 68, 381-388 (2018).

102. Kumar, A., Garg, R., Gaur, V. \& Kumar, P. Venlafaxine involves nitric oxide modulatory mechanism in experimental model of chronic behavior despair in mice. Brain Res. 1311, 73-80 (2010).

103. Varlinskaya, E. I., Johnson, J. M., Deak T. \& Diaz M. R. Adolescent forced swim stress increases social anxiety-like behaviors and alters the dynorphin/kappa opioid receptor system in the basolateral amygdala of males. bioRxiv 512350. https://doi.org/10.1101/512350 (2019).

104. Habr, S. F. Macrini, D. J., Spinosa, H. D. S. Florio, J. C. \& Bernardi, M. M. Repeated forced swim stress has additive effects in anxiety behavior and in cathecolamine levels of adult rats exposed to deltamethrin. Neurotoxicol. Teratol. 46, 57-61 (2014).

105. Shioda, N. et al. Dopamine D2L receptor deficiency causes stress vulnerability through 5-HT1A receptor dysfunction in serotonergic neurons. J. Neurosci. 39, 7551-7563 (2019).

106. Niknazar, S. et al. Comparison of the adulthood chronic stress effect on hippocampal BDNF signaling in male and female rats. Mol. Neurobiol. 53, 4026-4033 (2016).

107. Anuradha, H., Srikumar, B., Rao, B. S. \& Lakshmana, M. Euphorbia hirta reverses chronic stress-induced anxiety and mediates its action through the GABA A receptor benzodiazepine receptor-Cl-channel complex. J. Neural Transm. 115, 35-42 (2008).

108. Borsoi, M. et al. Immobility behavior during the forced swim test correlates with BNDF levels in the frontal cortex, but not with cognitive impairments. Physiol. Behav. 140, 79-88 (2015). 
109. Anderson, R. I., Lopez, M. F. \& Becker, H. C. Forced swim stress increases ethanol consumption in C57BL/6J mice with a history of chronic intermittent ethanol exposure. Psychopharmacology 233, 2035-2043 (2016).

110. Kondam, A., Kate, N. N. \& Lakshmi, G. Effect of forced swim stress on wistar albino rats in various behavioral parameters. Int. J. Med. Res. Health Sci. 1, 7-12 (2012).

111. Huang, Y. L., Chen, S. H., Su, Y. J. \& Kung, Y. W. Attachment dimensions and post-traumatic symptoms following interpersonal traumas versus impersonal traumas in young adults in Taiwan. Stress Health 33, 233-243 (2017).

112. Green, B. L. et al. Outcomes of single versus multiple trauma exposure in a screening sample. J. Trauma Stress. 13, 271-286 (2000).

113. Maercker, A., Beauducel, A. \& Schützwohl, M. Trauma severity and initial reactions as precipitating factors for posttraumatic stress symptoms and chronic dissociation in former political prisoners. J. Trauma Stress 13, 651-660 (2000)

114. Chen, Y. et al. Different regional gray matter loss in recent onset PTSD and non PTSD after a single prolonged trauma exposure. PLOS ONE 7, e48298 (2012).

115. Van der Kolk, B. A., Roth, S., Pelcovitz, D., Sunday, S. \& Spinazzola, J. Disorders of extreme stress: the empirical foundation of a complex adaptation to trauma. J. Trauma Stress 18, 389-399 (2005).

116. Overstreet, S. \& Mathews, T. Challenges associated with exposure to chronic trauma: using a public health framework to foster resilient outcomes among youth. Psychol. Sch. 48, 738-754 (2011).

117. Dorahy, M. J. et al. Complex PTSD, interpersonal trauma and relational consequences: findings from a treatment-receiving Northern Irish sample. J. Affect. Disord. 112, 71-80 (2009).

118. Gillespie, C. F. et al. Trauma exposure and stress-related disorders in inner city primary care patients. Gen. Hosp. Psychiatry 31, 505-514 (2009).

119. López, J. F., Chalmers, D. T., Little, K. Y. \& Watson, S. J. Regulation of serotonin1A, glucocorticoid, and mineralocorticoid receptor in rat and human hippocampus: implications for the neurobiology of depression. Biol. Psychiatry 43, 547-573 (1998).

120. Liu, Y., Beyer, A. \& Aebersold, R. On the dependency of cellular protein levels on mRNA abundance. Cell 165, 535-550 (2016).

121. Liu, D. et al. Changes in 5-HT1A receptor expression in the oculomotor nucleus in a rat model of post-traumatic stress disorder. J. Mol. Neurosci. $\mathbf{4 9}$ 360-368 (2013).

122. Xiang, M. et al. Stimulation of anxiety-like behavior via ERK pathway by competitive serotonin receptors $2 \mathrm{~A}$ and $1 \mathrm{~A}$ in post-traumatic stress disordered mice. Neurosignals 25, 39-53 (2017).

123. lyo, A. H. et al. Differential regulation of the serotonin 1 A transcriptional modulators five prime repressor element under dual repression-1 and nuclear-deformed epidermal autoregulatory factor by chronic stress. Neuroscience 163, 1119-1127 (2009).

124. Raghupathi, R. K. \& McGonigle, P. Differential effects of three acute stressors on the serotonin 5-HT1A receptor system in rat brain. Neuroendocrinology 65 , 246-258 (1997)

125. Kieran, N., Ou, X.-M. \& Iyo, A. H. Chronic social defeat downregulates the 5HT1A receptor but not Freud-1 or NUDR in the rat prefrontal cortex. Neurosci. Lett. 469, 380-384 (2010).

126. Szewczyk, B. et al. Stress-induced alterations in 5-HT1A receptor transcriptional modulators NUDR and Freud-1. Int J. Neuropsychopharmacol. 17, 1763-1775 (2014).

127. Rittenhouse, P. A. et al. Comparison of neuroendocrine and behavioral effects of ipsapirone, a 5-HT1A agonist, in three stress paradigms: immobilization, forced swim and conditioned fear. Brain Res. 580, 205-214 (1992).

128. Saphier, D., Farrar, G. E. \& Welch, J. E. Differential inhibition of stress-induced adrenocortical responses by $5-\mathrm{HT} 1 \mathrm{~A}$ agonists and by $5-\mathrm{HT} 2$ and $5-\mathrm{HT} 3$ antagonists. Psychoneuroendocrinology 20, 239-257 (1995).

129. Lin, L., Liu, G. \& Zhang, Y. Research on the effects of 5-HT1 A receptor in hippocampal CA1 region in cognition of PTSD rats. Adv. Eng. Res. 107, abstr. (2017).

130. Wang, L. et al. Serotonin signaling trough prelimbic 5-HT1A receptors modulates CSDS-induced behavioral changes in adult female voles. Int J. Neuropsychopharmacol. 22, 208-220 (2019).

131. Mendelson, S. D. \& McEwen, B. S. Autoradiographic analyses of the effects of restraint-induced stress on 5-HT1A, 5-HT1C and 5-HT2 receptors in the dorsal hippocampus of male and female rats. Neuroendocrinology 54, 454-461 (1991).
132. Newman-Tancredi, A., Albert, P. R., \& Sumiyoshi, T. In: Schizophrenia Research: Recent Advances 1st edn, Vol. 1 (ed Sumiyoshi, T.) Ch. 15 (Nova Science Publishers, Hauppauge, 2012).

133. Newman-Tancredi, A. Biased agonism at serotonin 5-HT1A receptors: preferential postsynaptic activity for improved therapy of CNS disorders. Neuropsychiatry 1, 149 (2011).

134. Assié, M. B., Lomenech, H., Ravailhe, V., Faucillon, V. \& Newman-Tancredi, A. Rapid desensitization of somatodendritic 5-HT1A receptors by chronic administration of the high-efficacy 5-HT1A agonist, F13714: a microdialysis study in the rat. Br. J. Pharmacol. 149, 170-178 (2006)

135. Assi, M.-B. et al. F15599, a highly selective post-synaptic 5-HT1A receptor agonist: in-vivo profile in behavioural models of antidepressant and serotonergic activity. Int. J. Neuropsychopharmacol. 13, 1285-1298 (2010).

136. Artigas, F., Celada, P. \& Bortolozzi, A. Can we increase the speed and efficacy of antidepressant treatments? Part II. Glutamatergic and RNA interference strategies. Eur. Neuropsychopharmacol. 28, 457-482 (2018).

137. Nemeroff, C. B. et al. Differential responses to psychotherapy versus pharmacotherapy in patients with chronic forms of major depression and childhood trauma. Proc. Natl Acad. Sci. 100, 14293-14296 (2003).

138. Nanni, V., Uher, R. \& Danese, A. Childhood maltreatment predicts unfavorable course of illness and treatment outcome in depression: a meta-analysis. Am J. Psychiatry 169, 141-151 (2012).

139. Shirk, S. R. \& Karver, M. Prediction of treatment outcome from relationship variables in child and adolescent therapy: a meta-analytic review. J. Consult Clin. Psychol. 71, 452 (2003)

140. Asarnow, J. R et al. Treatment of selective serotonin reuptake inhibitorresistant depression in adolescents: predictors and moderators of treatment response. J. Am. Acad. Child Adolesc. Psychiatry 48, 330-339 (2009).

141. Lewis, C. C. et al. Impact of childhood trauma on treatment outcome in the Treatment for Adolescents with Depression Study (TADS). J. Am. Acad. Child Adolesc. Psychiatry 49, 132-140 (2010).

142. Shamseddeen, $\mathbf{W}$. et al. Impact of physical and sexual abuse on treatment response in the Treatment of Resistant Depression in Adolescent Study (TORDIA). J. Am. Acad. Child Adolesc. Psychiatry 50, 293-301 (2011).

143. Shavitt, R. G. et al. The impact of trauma and post-traumatic stress disorder on the treatment response of patients with obsessive-compulsive disorder. Eur. Arch. Psychiatry Clin. Neurosci. 260, 91-99 (2010).

144. Williams, L. M., Debattista, C., Duchemin, A., Schatzberg, A. \& Nemeroff, C. Childhood trauma predicts antidepressant response in adults with majo depression: data from the randomized international study to predict optimized treatment for depression. Transl. Psychiatry 6, e799 (2016).

145. Rasheed, N. et al. Differential response of central dopaminergic system in acute and chronic unpredictable stress models in rats. Neurochem. Res. 35 22-32 (2010).

146. Yau, J. L., Noble, J. \& Seckl, J. R. Acute restraint stress increases 5-HT7 receptor mRNA expression in the rat hippocampus. Neurosci. Lett. 309, 141-144 (2001).

147. Yamamoto, M. et al. Effects of single and repeated prolonged stress on muopioid receptor mRNA expression in rat gross hypothalamic and midbrain homogenates. Brain Res. 980, 191-196 (2003).

148. Paskitti, M., McCreary, B. \& Herman, J. Stress regulation of adrenocorticosteroid receptor gene transcription and mRNA expression in rat hippocampus: time-course analysis. Mol. Brain Res. 80, 142-152 (2000).

149. McGowan, P. O. et al. Epigenetic regulation of the glucocorticoid receptor in human brain associates with childhood abuse. Nat. Neurosci. 12, 342 (2009).

150. Perroud, N. et al. Increased methylation of glucocorticoid receptor gene (NR3C1) in adults with a history of childhood maltreatment: a link with the severity and type of trauma. Transl. Psychiatry 1, e59 (2011).

151. Smith, A. K. et al. Differential immune system DNA methylation and cytokine regulation in post-traumatic stress disorder. Am. J. Med. Genet. B Neu ropsychiatr. Genet. 156, 700-708 (2011).

152. Breen, M. S. et al. PTSD blood transcriptome mega-analysis: shared inflammatory pathways across biological sex and modes of trauma. Neuropsychopharmacology 43, 469 (2018).

153. Mehta, D et al. Childhood maltreatment is associated with distinct genomic and epigenetic profiles in posttraumatic stress disorder. Proc. Natl Acad. Sci. 110, 8302-8307 (2013).

154. Rogaeva, A., Ou, X.-M., Jafar-Nejad, H., Lemonde, S. \& Albert, P. R. Differential repression by Freud-1/CC2D1A at a polymorphic site in the dopamine-D2 receptor gene. J. Biol. Chem. 282, 20897-20905 (2007). 
155. Huggenvik, J. I. et al. Characterization of a nuclear deformed epidermal autoregulatory factor-1 (DEAF-1)-related (NUDR) transcriptional regulator protein. Mol. Endorinol. 12, 1619-1639 (1998).

156. Jacobsen, K. X., Czesak, M., Deria, M., Le François, B. \& Albert, P. R. Regionspecific regulation of 5 -HT1A receptor expression by Pet-1-dependent mechanisms in vivo. J. Neurochem. 116, 1066-1076 (2011).

157. Fyodorov, D., Nelson, T. \& Deneris, E. Pet-1, a novel ETS domain factor that can activate neuronal nAchR gene transcription. J. Neurobiol. 34, 151-163 (1998).

158. Okamoto, S.-I., Sherman, K., Bai, G. \& Lipton, S. A. Effect of the ubiquitous transcription factors, SP1 and MAZ, on NMDA receptor subunit type 1 (NR1) expression during neuronal differentiation. Mol. Brain Res. 107, 89-96 (2002).

159. Parks, C. L. \& Shenk, T. The serotonin 1a receptor gene contains a TATA-less promoter that responds to MAZ and Sp1. J. Biol. Chem. 271, 4417-4430 (1996).

160. Lin, L., Liu, G. \& Sun, M. Effect of 5-HT1 A receptor in hippocampal CA1 region on spatial memory of PTSD rats. Chin. J. Pathophysiol. 33, 98-103 (2017)

161. Steciuk, M., Kram, M., Kramer, G. L. \& Petty, F. Acute stress does not alter 5HT1A receptor density. Prog. Neuropsychopharmacol. Biol. Psychiatry 24 155-161 (2000).

162. Fogaça, M. V., Reis, F., Campos, A. \& Guimarães, F. S. Effects of intra-prelimbic prefrontal cortex injection of cannabidiol on anxiety-like behavior: involvement of 5HT1A receptors and previous stressful experience. Eur. Neuropsychopharmacol. 24, 410-419 (2014).

163. Kim, M. H. \& Leem, Y. H. Chronic exercise improves repeated restraint stressinduced anxiety and depression through 5HT1A receptor and CAMP signaling in hippocampus. J. Exerc Nutr. Biochem. 18, 97 (2014).

164. Van Riel, E., Meijer, O., Steenbergen, P. \& Joels, M. Chronic unpredictable stress causes attenuation of serotonin responses in cornu ammonis 1 pyramidal neurons. Neuroscience 120, 649-658 (2003).

165. Ossowska, G. et al. Brain monoamine receptors in a chronic unpredictable stress model in rats. J. Neural Transm. 108, 311-319 (2001).

166. Watanabe, Y., Sakai, R. R., McEwen, B. S. \& Mendelson, S. Stress and antidepressant effects on hippocampal and cortical 5-HT1A and 5-HT2 receptors and transport sites for serotonin. Brain Res. 615, 87-94 (1993).

167. Ferretti, C., Blengio, M., Gamalero, S. R. \& Ghi, P. Biochemical and behaviour changes induced by acute stress in a chronic variate stress model of depression: the effect of amitriptyline. Eur. J. Pharmacol. $\mathbf{2 8 0}$ 19-26 (1995).

168. Flügge, G., Kramer, M., Rensing, S. \& Fuchs, E. 5HT1A-receptors and behaviour under chronic stress: selective counteraction by testosterone. Eur. J. Neurosci. 10, 2685-2693 (1998)

169. Morrison, K. E., Swallows, C. L. \& Cooper, M. A. Effects of dominance status on conditioned defeat and expression of 5-HT1A and 5-HT2A receptors. Physiol. Behav. 104, 283-920 (2011).

170. Ul'yana, A. B., Bondar, N. P., Filipenko, M. L. \& Kudryavtseva, N. N. Downregulation of serotonergic gene expression in the Raphe nuclei of the midbrain under chronic social defeat stress in male mice. Mol. Neurobiol. 48 13-21 (2013)

171. Cooper, M. A., Grober, M. S., Nicholas, C. \& Huhman, K. L. Aggressive encounters alter the activation of serotonergic neurons and the expression of 5-HT1A mRNA in the hamster dorsal raphe nucleus. Neuroscience 161 680-690 (2009).

172. Ohi, K., Mikuni, M. \& Takahashi, K. Stress adaptation and hypersensitivity in 5HT neuronal systems after repeated foot shock. Pharmacol. Biochem. Behav. 34, 603-608 (1989).

173. Campos, A. C., Ferreira, F. R. \& Guimarães, F. S. Cannabidiol blocks long-lasting behavioral consequences of predator threat stress: possible involvement of 5HT1A receptors. J. Psychiatr. Res. 46, 1501-1510 (2012).

174. Franklin, T. B., Linder, N., Russig, H., Thöny, B. \& Mansuy, I. M. Influence of early stress on social abilities and serotonergic functions across generations in mice. PloS ONE 6, e21842 (2011).

175. Maniam, J. \& Morris, M. J. Voluntary exercise and palatable high-fat diet both improve behavioural profile and stress responses in male rats exposed to early life stress: role of hippocampus. Psychoneuroendocrinology $\mathbf{3 5}$, 1553-1564 (2010).

176. Vázquez, D. M., Eskandari, R., Zimmer, C. A., Levine, S. \& López, J. F. Brain 5-HT receptor system in the stressed infant rat: implications for vulnerability to substance abuse. Psychoneuroendocrinology 27, 245-272 (2002).

177. Vicentic, A. et al. Maternal separation alters serotonergic transporter densities and serotonergic $1 \mathrm{~A}$ receptors in rat brain. Neuroscience $\mathbf{1 4 0}$, 355-365 (2006)

178. Li, M., Xue, X., Shao, S., Shao, F. \& Wang, W. Cognitive, emotional and neurochemical effects of repeated maternal separation in adolescent rats. Brain Res. 1518, 82-90 (2013).

179. Shishkina, G., Kalinina, T. \& Dygalo, N. Effects of swim stress and fluoxetine on 5-HT1A receptor gene expression and monoamine metabolism in the rat brain regions. Cell. Mol. Neurobiol. 32, 787-794 (2012).

180. Shishkina, G. T., Kalinina, T. S., Berezova, I. V. \& Dygalo, N. N. Stress-induced activation of the brainstem $\mathrm{BCl}-\mathrm{xL}$ gene expression in rats treated with fluoxetine: correlations with serotonin metabolism and depressive-like behavior. Neuropharmacology 62, 177-183 (2012).

181. Briones-Aranda, A., Rocha, L. \& Picazo, O. Influence of forced swimming stress on 5-HT1A receptors and serotonin levels in mouse brain. Prog. Neuropsychopharmacol. Biol. Psychiatry 29, 275-281 (2005).

182. Paré, W. P. \& Tejani-Butt, S. M. Effect of stress on the behavior and 5-HT system in Sprague-Dawley and Wistar Kyoto rat strains. Integr. Physiol. Behav. Sci. 31, 112-121 (1996).

183. Bravo, J. A., Dinan, T. G. \& Cryan, J. F. Early-life stress induces persistent alterations in 5-HT1A receptor and serotonin transporter mRNA expression in the adult rat brain. Front. Mol. Neurosci. 7, 24 (2014).

184. Van Riel, E., Van Gemert, N. G., Meijer, O. C. \& Joëls, M. Effect of early life stress on serotonin responses in the hippocampus of young adult rats. Synapse $\mathbf{5 3}$ 11-19 (2004).

185. Sullivan, G. M. et al. Positron emission tomography quantification of serotonin1A receptor binding in suicide attempters with major depressive disorder. JAMA Psychiatry 72, 169-178 (2015).

186. Festing, S. \& Wilkinson, R. The ethics of animal research. EMBO Rep. 8, 526-530 (2007).

187. Lemonde, S., Du, L., Bakish, D., Hrdina, P. \& Albert, P. R. Association of the C Chronic unpredictable stress causes attenuation of serotonin responses in cornu ammonis 1 pyramidal neurons(-1019) G 5-HT1A functional promoter polymorphism with antidepressant response. Internatio J. Neuropsychopharmacol. 7, 501-506 (2004). 